\title{
High-precision luminosity instrumentation for the CMS Experiment at the HL-LHC
}

\section{A. Ruede*}

European Organization for Nuclear Research (CERN), Geneva, Switzerland Institute for Data Processing and Electronics (KIT-IPE), Karlsruhe, Germany

E-mail: aruede@cern.ch

\section{G. Auzinger, A. Dabrowski}

European Organization for Nuclear Research (CERN), Geneva, Switzerland

\section{Oropeza Barrera}

Universidad Iberoamericana, Ciudad de Mexico, Mexico

\section{J. Cordero Mercado}

Northwestern University, Illinois, USA

The High Luminosity upgrade of the LHC (HL-LHC) is foreseen to increase the instantaneous luminosity by a factor of five over the present LHC nominal value. The resulting, unprecedented requirements for background monitoring and luminosity measurements create the need for new high-precision instrumentation at CMS, using radiation hard detector technologies.

This contribution discusses the implications of using the Tracker Endcap Pixel Detector for online measurements of luminosity and beam-induced background. The suitability of the TEPX detector for these tasks is explored and implementations for separate triggering and readout systems are explained.

Topical Workshop on Electronics for Particle Physics TWEPP2019

2-6 September 2019

Santiago de Compostela - Spain

\footnotetext{
*Speaker.
} 


\section{Introduction}

The future upgrade of the LHC accelerator at CERN, the High Luminosity LHC (HL-LHC), will increase its collision rate by a factor of five beyond the present nominal value. This allows a baseline operation of instantaneous peak luminosities of $5 \times 10^{34} \mathrm{~cm}^{-2} \mathrm{~s}^{-1}$ (with pileup of $\approx 140$ ), and an ultimate performance scenario of $7.5 \times 10^{34} \mathrm{~cm}^{-2} \mathrm{~s}^{-1}$ (with pileup of $\approx 200$ ) [1]. To ensure reliable luminosity and beam background measurements for the High Luminosity era of the CMS experiment, all the presently installed beam monitoring devices that are located in high radiation environments close to the beam pipe will need to be replaced due to radiation damage. The extreme radiation levels expected will not only require improved radiation-hardened technologies, but also sufficiently granular detectors to cope with the increased pileup.

This contribution presents the implications for using the Tracker Endcap Pixel Detector (TEPX) for online measurements of luminosity and beam-induced background. Section 2 describes the TEPX and its interesting characteristics for luminosity measurement. Supporting simulation results are presented in section 3 together with considerations for a dedicated luminosity trigger and the envisaged system design that allows luminosity measurement using the TEPX. A summary is provided in section 4 .

\section{The Tracker Endcap Pixel Detector}

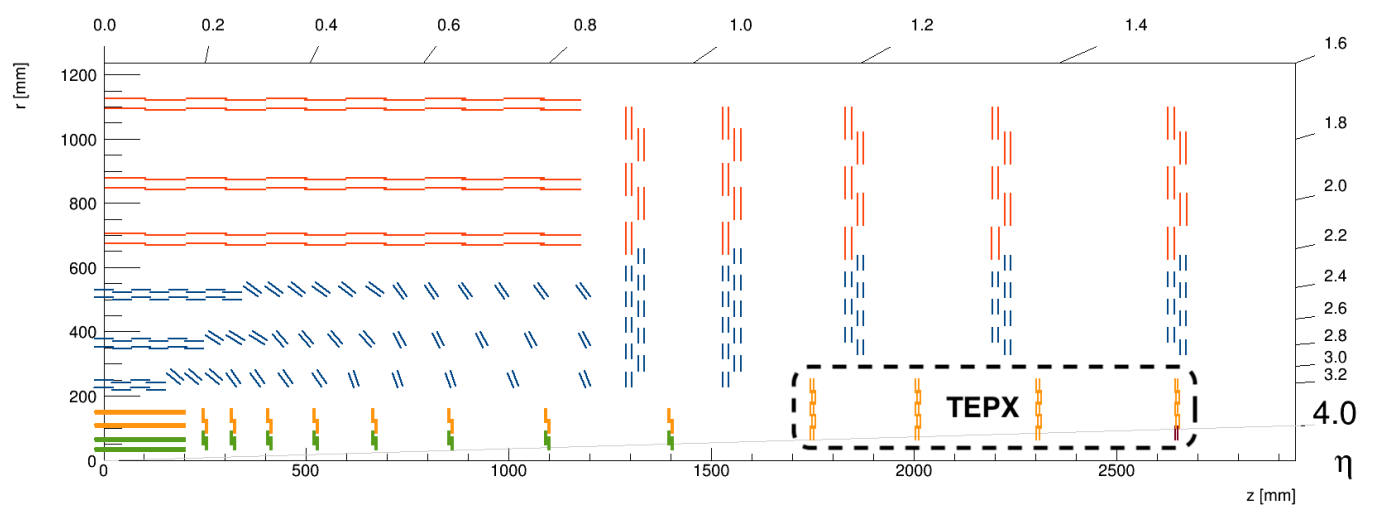

Figure 1: Proposed layout of the CMS Phase-II Tracker(OT614_200_IT613)

The upgrade of the CMS Tracker includes a forward extension of the Inner Tracker, the TEPX [2]. The TEPX will consist of four large double-disks per end, distributed between $|z| \approx 175 \mathrm{~cm}$ and $|z| \approx 265 \mathrm{~cm}$, as depicted in figure 1. Each double-disk is made of five rings equipped with $2 \times 2$ modules based on the RD53B pixel readout chip [3], which has been specifically designed for the ATLAS and CMS HL-LHC pixel detector upgrades.

Several characteristics of this detector make it a potentially very powerful instrument for online luminosity and beam-induced background (BIB) measurements in CMS. The RD53B readout chip will be produced in $65 \mathrm{~nm}$ CMOS technology and will be designed for a radiation tolerance up to 10 MGy. The position of the TEPX and its total area of about $2 \mathrm{~m}^{2}$ of silicon sensors allow highstatistics luminosity measurements with relatively low occupancy. Additionally, the z-position of 


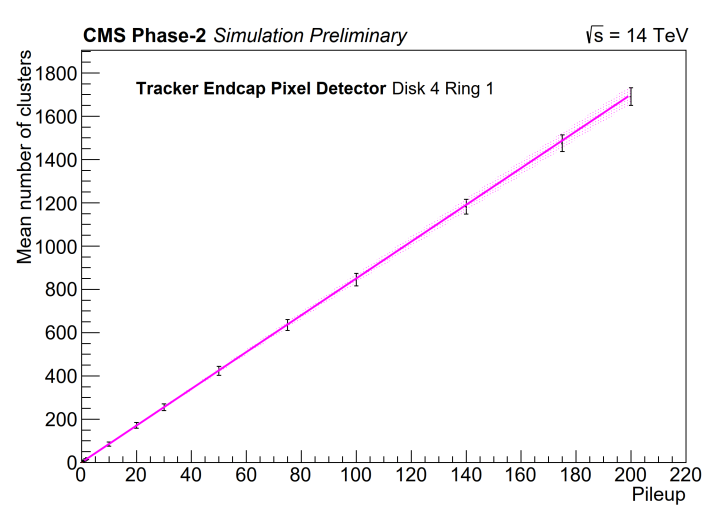

(a)

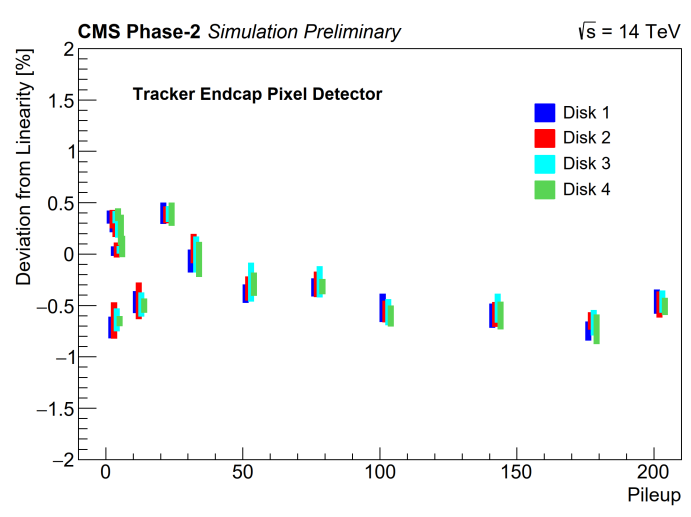

(b)

Figure 2: (a) Simulated mean number of clusters as a function of pileup for D4R1 of TEPX. (b) Relative non-linearity for all rings of all disks of TEPX.

the last double-disk (disk 4), about $2.65 \mathrm{~m}$ away from the interaction point (IP), is favourable for measuring the incoming BIB. At this location, the incoming bunch passes about $17 \mathrm{~ns}$ before the arrival of the outgoing collision products, allowing for an efficient background measurement in the first bunch crossing of each bunch train. Due to the high pseudorapidity $(|\eta|>4)$ of the innermost ring of the outermost disk, Disk 4 Ring 1 (D4R1), this ring is not needed for tracking and will serve as a dedicated detector for measuring luminosity and BIB.

\section{TEPX as Luminometer}

\subsection{Linearity Simulations}

Throughout previous runs the pixel cluster counting algorithm (PCC) [4] has been used for an offline measurement of the luminosity and achieved stability with low statistical error. This algorithm could be adapted for a real-time implementation in order to achieve a high precision online measurement of the luminosity.

To evaluate the luminosity measurement performance of cluster counting in TEPX, simulations have been carried out using the CMS software framework (CMSSW). Figure 2a shows the simulated mean number of clusters per event for TEPX D4R1 over a pileup range from 0 to 200. The result has been fitted in the interval from pileup 0 to 2 and extrapolated to pileup 200, to replicate the calibration process for luminometers during the so-called van der Meer scans [5]. The simulations show that the mean number of clusters is highly linear over the whole pileup range. The relative deviation from linear behaviour for each disk of TEPX as a band spanning the values from all rings is shown in figure $2 \mathrm{~b}$. This deviation is smaller than $1 \%$ over the full range of pileup for each ring and disk, rendering the TEPX a very suitable luminometer using the PCC algorithm.

\subsection{Luminosity Triggers}

The triggering requirements for luminosity differ substantially from the requirements that are driving the centrally managed CMS Level 1 (L1) trigger. For a luminosity measurement it is vital to 


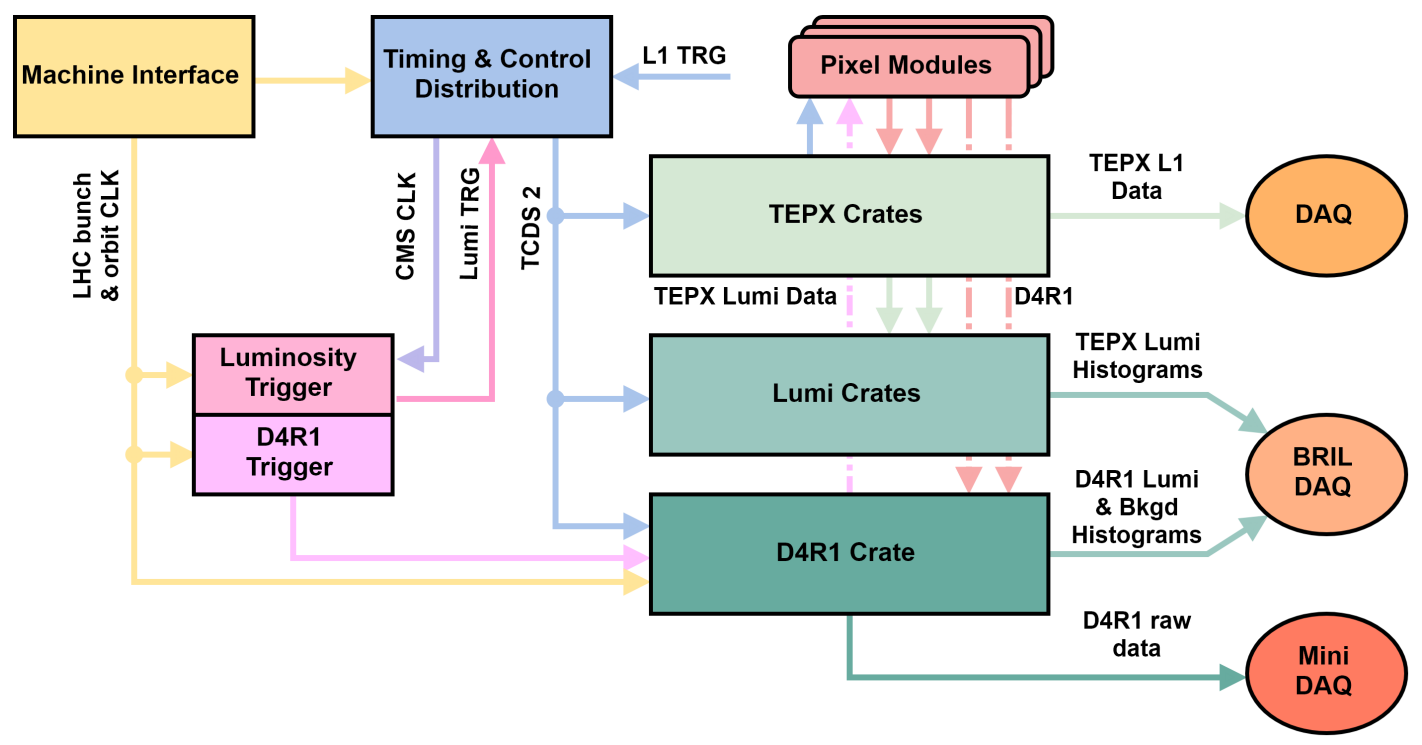

Figure 3: System overview of the TEPX detector (including D4R1) for the application as online luminosity- and background monitor. Control signal distribution and readout paths are shown for both TEPX and the independent D4R1.

provide an unbiased triggering scheme that equally samples all relevant bunch crossings in an orbit. Therefore, a dedicated luminosity trigger will be provided which implements a mix of zero-bias and random triggers and acts independently from the CMS L1 trigger.

During global CMS physics data taking a maximum trigger rate of $75 \mathrm{kHz}$ at a pileup of 200 will be allocated for luminosity in addition to the $750 \mathrm{kHz}$ L1 triggers. Outside of physics data taking, it is desirable to acquire luminosity data with a significantly higher rate. Particularly in special runs, an increased luminosity trigger rate is needed to achieve a high precision measurement.

The independent TEPX D4R1 does not need to accommodate the L1 trigger and thus can receive luminosity-specific triggers with a much higher rate. Since D4R1 will also be used for beam-induced background measurements, the triggering scheme might differ from the TEPX luminosity trigger. Therefore, an additional trigger specifically for D4R1 needs to be introduced.

\subsection{System Design}

In order to incorporate the luminosity measurement functionality in TEPX, the system has to be implemented such that the additional requirements are met. Figure 3 shows the system architecture that provides separate trigger generation and dedicated readout paths for the luminosity data from TEPX and from D4R1.

The luminosity triggers for TEPX are generated by a dedicated trigger board that has a bidirectional link to the master entity of the Timing and Control Distribution System (TCDS) [6] chain. These triggers are then distributed via the TCDS links to the back end system, which is based on the modular ATCA standard. The luminosity triggers will only be forwarded by the part of the back end that is interfacing with the TEPX pixel modules. The data that is read out from these modules will be separated according to their respective trigger type, so that the L1 data will be sent 
to the CMS central DAQ system. The luminosity data however, about $133 \mathrm{Gbps}$, will be sent via optical front panel links to a dedicated luminosity back end system, also based on ATCA technology. Here the data will be processed in real-time implementations of the PCC algorithms and other suitable algorithms for the determination of luminosity. The generated histograms are then sent to the dedicated BRILDAQ via the Gigabit Ethernet control network.

The trigger generation and back end system for D4R1 are completely separated from the rest of TEPX, in order to allow the measurement of the BIB even before the stable beams. Because the bunch and orbit clocks can be drifting during the beam energy ramp, the D4R1 back end system will be directly provided with these clocks. The triggers for D4R1, which will be based on a special scheme in order to provide BIB measurement, will not be distributed by TCDS, but will be generated and distributed in a local stream that resembles the TCDS stream. The D4R1 pixel modules will be interfacing with the dedicated D4R1 back end system for readout and data processing, only honouring the D4R1 triggers. In order to be able to occasionally save full pixel event data from D4R1 for calibration purposes and offline studies, this part of the system should be connected to a so-called miniDAQ instance, which is a fully featured, but separate instance of the CMS central DAQ that is configured and operated independently from global data taking. This is necessary as the requirement for different clocks during ramp mode prevents D4R1 data from being synchronized with the rest of CMS and thus preventing D4R1 to send data to the central DAQ.

\section{Summary}

The challenging requirements for high-precision luminosity measurements in CMS for the high luminosity era of the LHC can be met by exploiting the Tracker Endcap Pixel Detector (TEPX). By implementing dedicated and separate triggering and back-end systems, it is possible to provide a reliable luminosity measurement without interfering with the physics objective of the pixel detector. Additionally, the presented system architecture allows the ring 1 of disk 4 of TEPX to serve as a standalone detector for luminosity and beam-induced background measurements.

\section{References}

[1] G. Apollinari et al., High-Luminosity Large Hadron Collider (HL-LHC): Preliminary Design Report, CERN-2015-005, doi: 10.5170/CERN-2015-005, 2015.

[2] CMS Collaboration, The Phase-II Upgrade of the CMS Tracker, CERN-LHCC-2017-009. CMS-TDR-014, 2017.

[3] RD53 Collaboration, RD53B Design Requirements, CERN-RD53-PUB-19-001, 2019.

[4] CMS Collaboration, CMS luminosity measurement for the 2016 data taking period, CMS Physics Analysis Summary CMS-PAS-LUM-17-001, 2017.

[5] S. van der Meer, Calibration of the effective beam height in the ISR, Technical Report CERN-ISR-PO-68-31, 1968.

[6] J-M Andre et al, The CMS Data Acquisition - Architectures for thePhase-2 Upgrade, J. Phys.: Conf. Ser. 898 032019, 2017. 